\title{
A Comparison Between Commercial and Open-Source Software for Finite Element Analysis of Elasto-Plastic Bending
}

\author{
Ahmed Abdelaty*, Christian Reisinger, Christian Zehetner \\ University of Applied Sciences Upper Austria \\ Stelzhamerstraße 23, 4600 Wels, Austria \\ Email: ahmed.abdelaty@students.fh-wels.at*,christian.zehetner@fh-wels.at \\ web page: http://www.fh-ooe.at
}

\begin{abstract}
Nowadays, simulation is becoming more and more important in industries. Here we consider a typical industrial application in the field of sheet metal bending machines. On the one hand, a high number of simulations is necessary during the development process, e.g., to perform parameter studies and optimizations. On the other hand, simulation tools should be also available for the customers, e.g., to plan the production of very specific profiles. In such cases, the optimal process parameters only can be found by simulation.

A very critical point in this context are the license costs for commercial simulation software. Frequently, the simulations are not limited by computational power but by the number of available licenses, such that the duration for parameter studies is elongated. Also, with license costs it is not possible to provide a simulation platform to the customers.

The presented case study has been carried out with the goal of comparing possible alternatives to the expensive license for finite element analysis platforms. For comparison, we consider the elasto-plastic bending of a cantilever: a two-dimensional plane strain analysis (2-D model), as well as a stress-strain analysis based on three-dimensional continuum elements (3-D model). The results of open-source software (Salome Meca) are compared to a commercial counterpart (Abaqus).

The models are used as a basis for a case study to compare the performance of both platforms using different matched cases and various material models such as linear elastic or nonlinear elasto-plastic models, e.g. the Johnson-Cook model [1][2]. The comparison covers aspects regarding the usability of each platform, including the time required to build and run a simulation, solver properties, troubleshooting errors, viewing and rendering of results.

Considering the obvious price difference, both platforms show comparable results. In addition, using Salome Meca that can run natively on Linux has the benefit of running on cloud servers to run highly demanding computations. Also, there is the possibility to run it on a virtual machine on other operating systems. Comparing the functionality of both programs, both are capable of modelling highly detailed and complex models for elasto-plastic material processing. However, for understanding the structure of the user interface of Salome Meca is far more time consuming. All in all, it turned out that depending on the specific application Salome Meca can be a powerful alternative to Abaqus for the considered industrial application.
\end{abstract}

\section{REFERENCES}

[1] Zehetner, C., Reisinger, C., Kunze, W., Hammelmüller, F., Eder, R., Holl, H., \& Irschik, H. (2021). Highquality sheet metal production using a model-based adaptive approach. Procedia Computer Science, 180, 249-258. https://doi.org/10.1016/j.procs.2021.01.162

[2] Johnson, G. R., \& Cook, W. H. (1985). Fracture characteristics of three metals subjected to various strains, strain rates, temperatures and pressures. Engineering Fracture Mechanics, 21(1), 31-48. https://doi.org/10.1016/0013-7944(85)90052-9 